\title{
106. The Assignment of the Nucleolar Organizer in the Chromosomes of the Funa (Carassius, Cyprinidae, Pisces)
}

\author{
By Yoshio OJIma and Takashi Yamano \\ Department of Biology, Faculty of Science, Kwansei Gakuin \\ University, Nishinomiya
}

(Communicated by Sajiro Makino, M. J. A., Nov. 12, 1980)

Following the quinacrine fluorescence $(\mathrm{Q})$ banding method established by Caspersson et al. (1968), several other Giemsa staining banding techniques (G-, C-, R- and others) had developed: They served as useful tools for efficient and precise analyses of chromosomes in various animals beyond the routine Giemsa method level and had promoted the development of cytogenetics of animals to a great extent. The characterization of chromosomes in mammals as well as in other animals has been extremely refined through the application of these procedure. Recently, the silver-staining technique was developed for the demonstration of the nucleolus organizer regions (NORs) in mammalian chromosomes. Howell and Black (1978) reported that visible NORs were defined in the fish, Fundulus diaphanus, in spite of the fact that their chromosomes are relatively tiny in general morphology. Excited by the work of Howell and Black (1978), the authors have undertaken a comparative study of chromosomes with special regard to the NORs in the Japanese Funa, Gold-fish and the Chinese Funa.

There is a considerable discrepancy in the systematic classification of the Japanese Funa (Carassius auratus), particularly because of their polymorphic distributional features. The Japanese Funa has been subdivided according to their distribution into the following five subspecies: Carassius a. subsp.; C. a. langsdorfii; C. a. grandoculis; C. a. buergeri; and C. a. cuvieri. The chromosome of Carassius a. cuvieri were reported as $2 n, 100$ in both sexes (Ojima et al., 1966; Ojima and Hitotsumachi, 1967), and the same condition was established in C. a. subsp. from Lake Kasumigaura (Kobayashi et al., 1970, 1973). Also C. a. langsdorfii from Lake Shinji had 2n, 100 in both sexes (Kobayashi et al., 1970, 1973). Carassius a. langsdorfii from Miyazaki district has been known to be in the same condition in their sexuality and chromosome number. In contrast, the occurrence of specimens characterized by the chromosome numbers in $3 n$ - and $4 n$ ranges has been found in those from Kanto district (Kobayashi et al., 
1970). Remarkable is the fact that the $3 n$-individuals of C. a. langsdorfii propagate unisexually by gynogenesis (Kobayashi et al., 1970, 1972). Ojima and Asano (1977) informed that the population of $C$. a. langsdorfii occurring in the Lake of Biwa generally consisted of females with $3 n$-chromosomes and bred by gynogenesis. A lot of similarities were found between $C$. a. buergeri and C. a. langsdorfi with regard to their sexuality and chromosome numbers. Carassius $a$. buergeri from Lake Suwa showed a 50:50 sex-ratio together with the chromosome number of $2 n, 100$, in both sexes. The Hokkaido population consisted, however, of female specimens characterized by $3 n$ chromosomes with gynogenetic reproduction (Kobayashi et al., 1973). According to Muramoto (1975), the triploid-range specimens would be found not only in both sexes of $C$. a. langsdorfii but also in $C$. a. cuvieri.

Materials and methods. Carassius a. subsp., 우 2 and $\$ 3$, were collected from Lake Jonuma in Gunma Prefecture, and 2 females and 2 unknown sex individuals of $C$. a. buergeri were obtained from the Lake of Suwa, Nagano Prefecture. Further, 3 females of $C$. a. langsdorfii, 1 female and 1 male of $C$. a. grandoculis, and 2 females and 3 males of $C$. a. cuvieri were collected from Lake Biwa, Shiga Prefecture. In addition, 6 unknown sex individuals of Wakin (Carassius a. auratus) were obtained in the market, and 1 female and 2 males of the Chinese Funa (Carassius a. auratus) came from China. They provided materials for the present investigations. The chromosome preparations were made according to the direct method based on the kidney tissue, as well as on the kidney cultures (Yamamoto and Ojima, 1973). In addition epithelial cells from fins were availably used for study (Hayashi and Ojima, 1976). Following the colchicine treatment $(0.5 \mu \mathrm{g} / \mathrm{ml})$ for $30 \mathrm{~min}$ at room temperature, KCl-hypotonization (0.075 M) for 15-20 min and Carnoy-fixation as usual, the Giemsa staining was applied to the slides.

After microscopic observations, the slides were differentiated by $90 \%$ alcohol. The NORs staining was processed according to a modification of the $\mathrm{Ag}$-As staining method by Goodpasture and Bloom (1975), and by Klingerman and Bloom (1977).

Three kinds of solutions, such as (1) Ag solution of $50 \%$ aqueous $\mathrm{AgNO}_{3}$, (2) an ammoniacal silver (As) solution prepared by dissolving $4 \mathrm{~g} \mathrm{AgNO}_{3}$ in a solution of 5cc deionized water and 5cc concentrated ammonium hydroxide, (3) developing solution of acetate-buffered $3 \%$ formalin adjusted to $\mathrm{pH} 4.2$, were prepared for staining. The Ag solution was pipetted onto a slide, covered with a coverglass and incubated at $50^{\circ} \mathrm{C}$ for $20 \mathrm{~min}$. The coverglass was rinsed off by deionized water and air-dried. The slides were developed by adding 
4 drops of As solution followed by 4 drops of developing solution, covered with coverslips. After proper development, the coverslips were rinsed off in deionized water and the slides were air-dried.

Results and remarks. Carassius a. subsp., C. a. buergeri, C. a. grandoculis, C.a.cuvieri, Wakin (C.a.auratus) and the Chinese Funa (C. a. auratus) were all characterized by $2 n=100$, consisting of six pairs of metacentrics, eighteen pairs of submetacentrics and twentysix pairs of subtelo-acrocentrics (Figs. 1-2). A comparison of karyotypes of C. a. cuvieri, the Wakin and Ryukin in the previous paper (Ojima et al. 1966) has failed to reveal any apparent change or variation of individual chromosomes, either numerically or morphologically.

As shown in Figs. 1-2, the areas of intense silver deposition (AgNORs) were constantly and commonly seen to deal with the short arms of the 12th largest submetacentric chromosomes in the subspecies of the Funa as mentioned above. Howell and Black (1979) noted that heavily stained NORs occurred on the short arms of the sex chromosomes of both males and females. Occasionally, tiny NORs sites could be seen over the secondary constrictions of the chromosome pair 3. In the present investigations, no remarkable sexual differences of the NORs was detected in the specimens under study. For understanding of sex determination in teleosts the following items of elucidation would be required: (1) The cytogenetic mechanism, (2) morphological process, and (3) hormonal control. In teleosts, however, our knowledge has remained very poorly on these aspects in comparison with other animals (Vanyakia, 1972). On the basis of a consensus of estimates through the facts and views as mentioned above, the statements may be available that the fish group is now being in the course of evolution, and that the sexual differentiation is still in an unstable condition.

Fig. 3 is the karyotype of C. a. langsdorfii from Lake Biwa, which is characterized by a pair of NORs on the middle part of submetacentric chromosomes, and on several other tiny chromosomes.

Ueda and Ojima (1978) investigated comparatively the C-banding patterns of chromosomes in five subspecies of the Funa (Carassius auratus), and resulted in that C. a. langsdorfii which was gynogenetic with a triploid complement, was remarkable by having a pair of markers characterized by outstanding C-bands on the short arms of the second largest submetacentrics. In $C$. $a$. subsp., there were two C-banded markers in the female complement while one in the male. The markers as found in C. a. subsp., however, could not be demonstrated in several other subspecies of the Funa such as C. a. cuvieri, C. a. grandoculis, and C. a. buergeri. Ojima et al. (1979) found that 


\section{CP 8888888888 \\ fit}

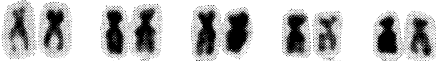

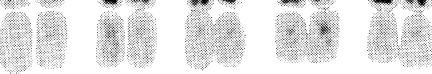

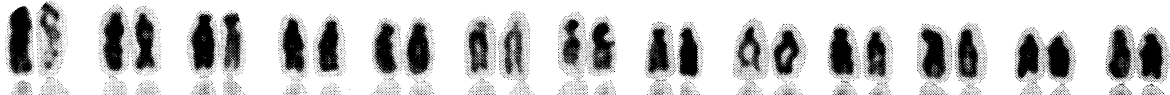

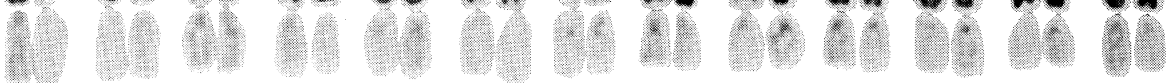

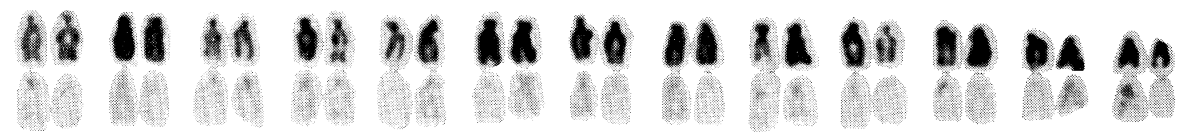

$8 \times 8888 \times 2 \times 8 \times 2 \quad 5 \mu \quad 2$

8. (2.

do

17) 88 1: 11 a1

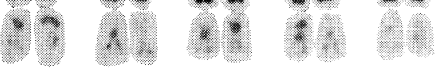

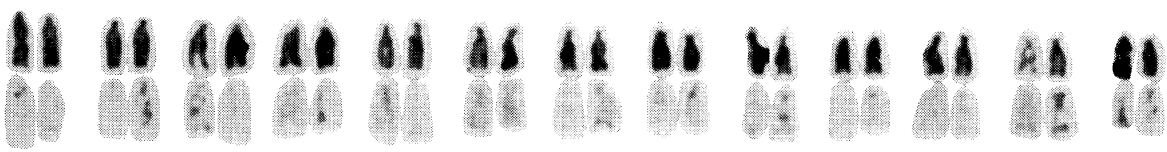

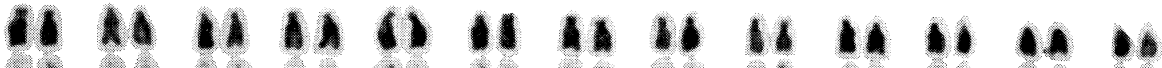

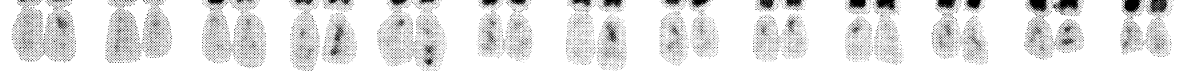

Figs. 1-2. 1: Karyotype of all varieties in Funa and Gold-fish with 100 chromosomes in diploid (the upper row). The arrows in the lower row denote 12th largest submetacentrics having intensely Ag-NORs. 2: Karyotype and Ag-NORs (arrows) in the Chinese Funa (C. a. auratus), showing as the same constitution as Fig. 1. 
the varieties of Gold-fish except the Shubunkin and the Comet were characterized by the second largest submetacentric marker chromosomes carrying C-bands on their short arms, being two in the females and one in the males. Under the comparison with the present NORs patterns, the following conclusion seems to be allowed that there are no significant correlations between C-banding patterns and NORs regions in the Funa and Gold-fish chromosomes.

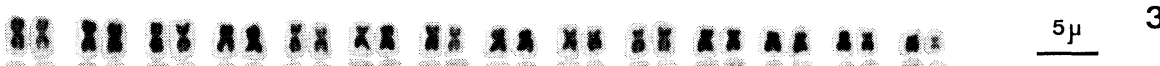

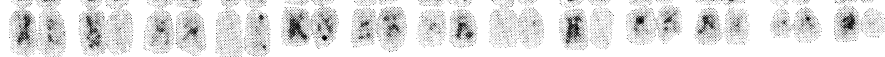

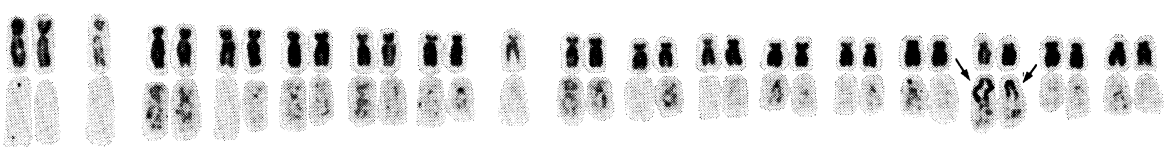

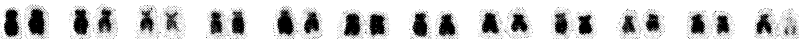

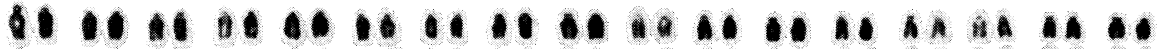

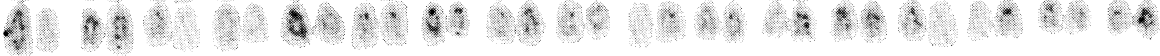

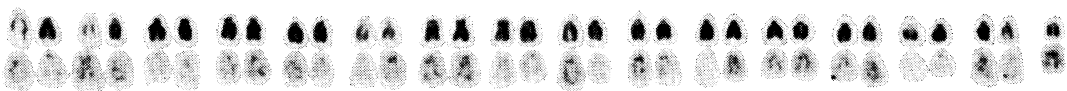

Fig. 3. Karyotype (the upper row) and Ag-NORs in C. a. langsdorfii with $3 n$-range chromosomes having two intensely Ag-NORs among the mediansized submetacentrics and several tiny chromosomes (arrows).

Summary. In six subspecies of Carassius auratus and Gold-fish including Carassius a. subsp., C. a. buergeri, C. a. grandoculis, C. a. cuvieri, C. a. langsdorfii, the Chinese Funa (C. a. auratus) and the Wakin (C. a. auratus), the NORs patterns of chromosomes were comparatively investigated. The areas of intense silver deposition (AgNORs) were constantly and commonly associated with the short arms of the 12th largest submetacentric chromosomes in the varieties except $C$. a. langsdorfii. In C. a. langsdorfii there were two such elements among the median-sized submetacentric chromosomes. No remarkable sexual differences of the NORs was detected.

Acknowledgement. The present authors are cordially indebted to Dr. Sajiro Makino, M. J. A., Professor Emeritus of Hokkaido University, for refinement of the manuscript with critical revision of data.

\section{References}

Caspersson, T., Farber, S., Foley, G. E., Kudynowski, J., Modest, B. J., Simonsson, E., Wagh, U., and Zech, L. (1968) : Expl. Cell Res., 49, 219-222. 
Goodpasture, C., and Bloom, S. E. (1975) : Chromosoma, 53, 37-50.

Hayashi, M., Ojima, Y., and Asano, N. (1976) : Japan. J. Genet., 51, 65-68.

Howell, W. M., and Black, D. A. (1979) : Copeia, 1979, no. 3, pp. 544-546.

Kobayashi, H., Kawashima, Y., and Takeuchi, N. (1970): Japan. J. Ichthyol., 17, 153-160.

Kobayashi, H. (1971) : Zool. Mag., 81, 67-71.

Kobayashi, H., and Ochi, H. (1972) : Zool. Mag., 81, 67-71.

Kobayashi, H., Ochi, H., and Takeuchi, N. (1973) : Japan. J. Ichthyol., 20, 7-12.

Klingerman, A. D., and Bloom, S. E. (1979) : J. Fish. Res. Board Can., 34, 266269.

Muramoto, J. (1975) : Proc. Japan Acad., 51, 583-587.

Ojima, Y., Hitotsumachi, S., and Makino, S. (1966): Proc. Japan Acad., 42, 163-167.

Ojima, Y., and Hitotsumachi, S. (1977) : Japan. J. Genet., 42, 163-167.

Ojima, Y., and Asano, N. (1977) : Proc. Japan Acad., 53, 138-142.

Ojima, Y., Ueda, T., and Narikawa, T. (1979) : Proc. Japan Acad., 55B, 58-63.

Ueda, T., and Ojima, Y. (1978) : Proc. Japan Acad., 54B, 283-288.

Vanyakia, E. D. (1972): Genetics, Selection and Hybridization of Fish (ed. Cherfas, B. I.). Israel Program for Scientific Translations Ltd., Jersalem, pp. $5-24$.

Yamamoto, K., and Ojima, Y. (1973) : Japan. J. Genet., 48, 235-238. 